\title{
New 3D Graphics Rendering Engine Architecture for Direct Tessellation of Spline Surfaces
}

\author{
Adrian Sfarti, Brian A. Barsky, Todd J. Kosloff, Egon Pasztor, \\ Alex Kozlowski, Eric Roman, and Alex Perelman \\ University of California, Berkeley
}

\begin{abstract}
In current 3D graphics architectures, the bus between the triangle server and the rendering engine GPU is clogged with triangle vertices and their many attributes (normal vectors, colors, texture coordinates). We develop a new 3D graphics architecture using data compression to unclog the bus between the triangle server and the rendering engine. The data compression is achieved by replacing the conventional idea of a GPU that renders triangles with a GPU that tessellates surface patches into triangles.
\end{abstract}

\section{Introduction}

The main goal of this paper is to develop a new graphics architecture that exploits faster arithmetic such that the CPU will serve parametric patches and the rendering engine (GPU) will triangulate these patches in real time.

The proposed architecture handles freeform parametric rational and non-rational spline surfaces such as Bézier and B-spline/NURBS. In comparison with current graphics architectures, which are based on transferring triangle vertices, the amount of data sent over the bus between the CPU and the GPU will be reduced by a factor that is linear in the number of triangles forming each surface patch. That is, if a surface patch is tessellated into $\mathrm{n}$ triangles, then the bus bandwidth required is $1 / \mathrm{n}$ of the bandwidth that would have been required by the conventional method of transferring triangle vertices. Since this approach stores control points of the surface patch instead of triangle vertices, the memory footprint will also be reduced by this same factor. For example, today's top of the line GPUs are capable of rendering about 500 million meshed triangles per second. At about 30 bytes/vertex (color, texture coordinates, geometry, normals) this results into a bus bandwidth requirement of about $15 \mathrm{Gbytes} / \mathrm{sec}$ ond while the fastest buses available have a peak bandwidth about 4 times lower.

Since today's Transformation Units are programmable processors, we envisage our implementation not as a silicon implementation but rather as reprogramming an already existent Transformation Unit inside an already existent GPU. Our simulations show that due to the reduced transformation effort (we replace the transformation of the triangle vertices with the transformation of the surface control points) we can easily reprogram one of the Transformation Units to act as a Tesselator Unit. 

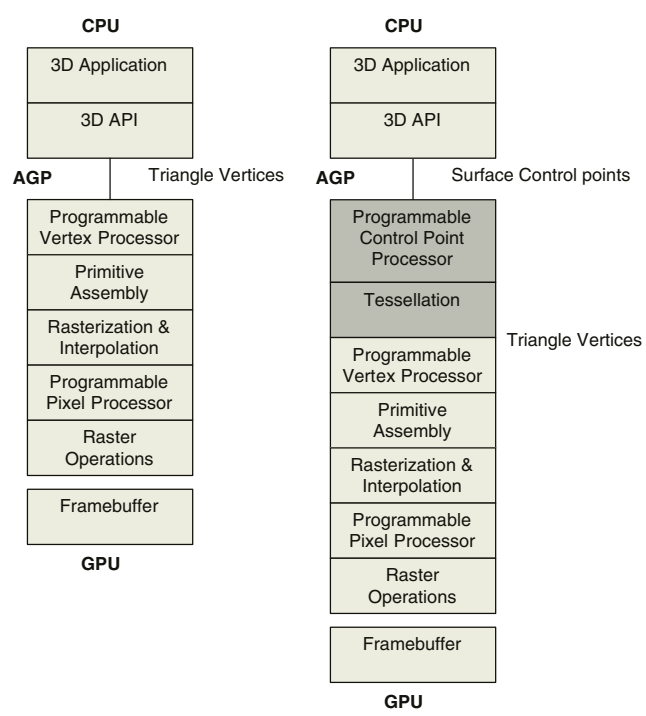

Fig. 1. Conventional programmable architecture (left), new architecture (right). The new architecture adds two stages to the GPU pipeline, which are shown in grey

\section{Previous Work}

There have been very few implementations of real time tesselation in hardware. In the mid1980's, Sun developed an architecture for this that was described in [1] and in a series of associated patents. The implementation was not a significant technical or commercial success because it did not exploit triangle based rendering; instead it attempted to render the surfaces in a pixel-by-pixel manner [2]. The idea was to use adaptive forward differencing to interpolate infinitesimally close parallel cubic curves imbedded into the bicubic surface.

More recently, Henry Moreton from NVIDIA has resurrected the real time tesselation unit [3]. This method does not directly tesselate patches in real time; rather, it uses off-line pre-tesselated triangle meshes in conjunction with a proprietary stitching method that avoids cracking and popping at the seams. Using this approach, the tessellation unit exists in front of the transformation unit and outputs triangle databases to be rendered by the existent components of the $3 \mathrm{D}$ graphics hardware.

The current paper is based on a recent patent [4] that is the first to introduce a real time tesselation processor into a GPU pipeline. To date, there is no GPU built with a real time tesselator processor but we hope that the current article will spark the design of such a device.

\section{GPU Architectures}

\subsection{Current State of the Art}

The pseudo code describing the current state of the art GPU architectures is shown below for the bicubic case. Notation: Let $\left(s_{i}, t_{j}\right)$ denote a pair of parameter values used 
in patch parameterization, $V_{i, j}$ a vertex, and $N_{i, j}$ a vertex normal. Also, we denote texture coordinates by $u_{i, j}$ and $v_{i, j}$.

Step 1. (off-line). For each bicubic surface, subdivide the $S$ and $T$ intervals until each resultant four-sided surface is below a certain predetermined curvature value.

Step 2. For all bicubic surfaces sharing a common boundary, take the union of the subdivisions to prevent cracks along the common boundary.

Step 3. For each bicubic surface, For each pair $\left(s_{i}, t_{j}\right)$, Calculate $\left(u_{i, j}, v_{i, j}, q_{i, j}, V_{i, j}\right)$, Generate triangles by connecting neighboring vertices.

Step 4. For each vertex $V_{i, j}$ Calculate the normal $N_{i, j}$ to that vertex (used for lighting). For each triangle Calculate the normal to the triangle (used for culling).

Step 5. (real time). Transform the vertices $V_{i, j}$ and the normals $N_{i, j}$ and the normals to the triangles. For each vertex $V_{i, j}$, Calculate lighting.

\subsection{The Tesselator Unit - Principles of Operation}

Though we have not implemented our proposed GPU in silicon yet we are publishing below the behavioral Verilog code describing the principles of operation. From this behavioral code a silicon implementation of the proposed GPU architecture should be straightforward:

Step 0 (all steps in real time). For each bicubic surface Transform the 16 control points that determine the surface.

Step 1. For each bicubic surface, Subdivide the boundary curve representing the $s$ interval until the projection of the length of the height of the curve bounding box is below a certain predetermined number of pixels as measured in screen coordinates. Repeat the process for the boundary curve representing the $t$ interval. For each new view, a new subdivision can be generated, producing automatic level of detail.

Step 2. For all bicubic surfaces sharing a same parameter (either $s$ or $t$ ) boundary, Choose as the common subdivision the reunion of the subdivisions in order to prevent cracks showing along the common boundary OR choose as the common subdivision the finest subdivision (the one with the most points inside the set) OR insert a zippering strip to smoothly progress from one patch to its neighbor. (Prevent cracks at the boundary between surfaces).

Step 3. (Generate the vertices, normals, the texture coordinates and the displacements used for bump and displacement mapping for the present subdivision) For each bicubic surface, For each pair $\left(s_{i}, t_{j}\right)$ Calculate $\left(\left(u_{i, j}, v_{i, j}, q_{i, j}\right),\left(p_{i, j}, r_{i, j}\right), V_{i, j}\right)$ through interpolation (texture, displacement map and vertex coordinates as a function of $\left(s_{i}, t_{j}\right)$ ) Look up vertex displacement $\left(d x_{i, j}, d y_{i, j}, d z_{i, j}\right)$. Generate triangles by connecting neighboring vertices.

Step 4. For each vertex $V_{i, j}$ Calculate the normal $N_{i, j}$ to that vertex. 


\section{The Subdivision Step}

We use the Lane-Carpenter subdivision algorithm described in [5] but we apply our own termination criterion. The four boundary curves of a Bézier patch are themselves Bézier curves, which we subdivide using the de Casteljau formulas. The edge subdivision results in a subdivision of the parametric intervals These parameter values are stored, whereas the control points resulting from subdivision are discarded immediately after the termination test is run. After the subdivision and crack prevention steps, the actual vertex locations throughout the patch are computed from the stored parameter values. The generation of vertices is comparable with vertex transformation. Note that the vertices are generated already transformed in place because the parent bicubic surface has already been transformed.

\section{Termination Criteria}

Our algorithm decides that an edge curve has been sufficiently subdivided when the trapezoidal convex hull of that curve has a sufficiently small height, as seen from the viewpoint of the observer. Referring to Figure 2, subdivision terminates when the following conditions are met:

$$
\begin{aligned}
& \operatorname{Max}\left\{\operatorname{dist}\left(P_{12} \text { to line }\left(P_{11}, P_{14}\right)\right), \operatorname{dist}\left(P_{13} \text { to line }\left(P_{11}, P_{14}\right)\right)\right\} \times \frac{2 d}{\left(\left|P_{12 z}\right|+\left|P_{13 z}\right|\right.}<n \text { AND } \\
& \operatorname{Max}\left\{\operatorname{dist}\left(P_{24} \text { to line }\left(P_{14}, P_{44}\right)\right), \operatorname{dist}\left(P_{34} \text { to line }\left(P_{14}, P_{44}\right)\right)\right\} \times \frac{2 d}{\left(\left|P_{24 z}\right|+\left|P_{34 z}\right|\right)}<n
\end{aligned}
$$

where $n$ is an arbitrary number expressed in pixels or in a fraction of pixels and $d$ is the distance from the viewer to the projection plane.

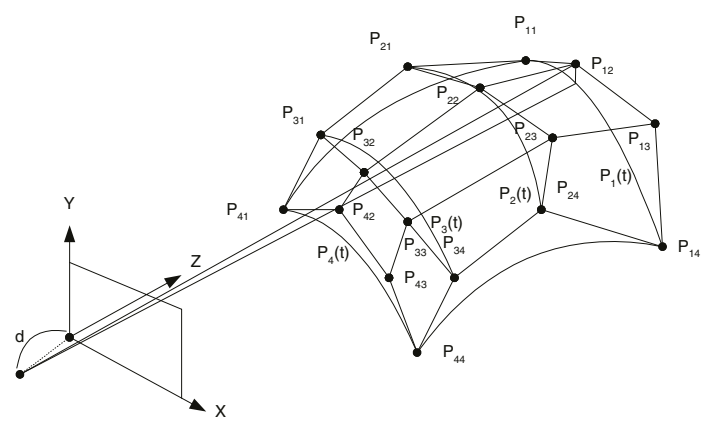

Fig. 2. Termination Criteria

We experimented with $\mathrm{n}$ starting at 1 and we observed that there were artifacts, especially along the silhouette. Forsey et al. [6] seem to settle on $n=.5$ and we tried that. We also experimented with $n>1$, for reasons of rapid prototyping and previewing. 
The above criterion is sufficient for surface patches that are not more curved inside their boundaries than they are along their boundaries. Conversely, if the patches are more curved inside than they are along their boundaries, we terminate subdivision based on the internal curves of the patch.

Since the curvature of free-form surfaces can switch between being boundary-limited and internally-limited, we will need to measure the flatness of both types of curves at the start of the tesselation associated with each instance of the surface by subdividing the four boundary curves as well as two orthogonal internal curves, specifically the curves that interverne in the second termination criterion shown above. We can further exploit the fact that adjacent patches share two boundary curves so that we need to subdivide only two of the four boundary curves for each patch. The only obvious exceptions are the patches at the boundary of a surface, since such patches have fewer than four neighbors. In the case of the boundary patches, our algorithm always subdivides all four boundary curves.

Our termination test ensures that patches are subdivided sufficiently to avoid silhouette artifacts. However, the test was shown to be insufficient in the case of a large flat patch facing the viewer. Such a patch would not be subdivided because a single pair of triangles can completely capture the geometry of this curve. However, per-vertex lighting would lead to highlight artifacts. Additionally, nearly-flat portions of a patch exhibit undesirable texture flickering as they visibly transition from one level of detail to the next. This is because the bilinear texture coordinate interpolation that we use when assigning texture coordinates to triangle vertices is not the same as the method used to interpolate within a triangle. To combat this problem, we decree that patches must be subdivided a minimum number of times, regardless of curvature.

\section{Crack Prevention}

If there are no special prevention methods, cracks may appear at the boundary between abutting patches. This is mainly due to the fact that the patches are subdivided independently of each other. Abutting patches can exhibit different curvatures resulting in different subdivisions. When rendering the parallel strips of triangles to the left and to the right of the common boundary, a crack may become visible.

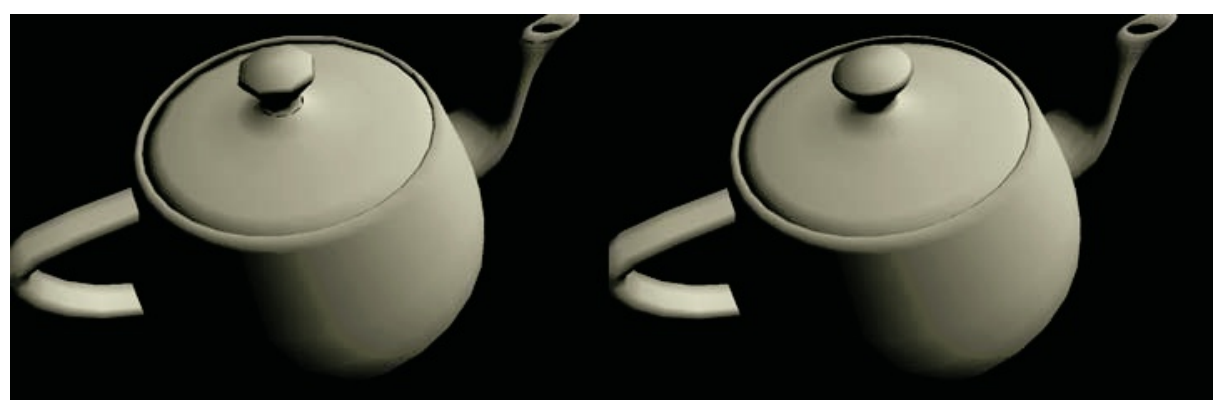

Fig. 3. Left: Without Crack Prevention. Right: With Crack Prevention. 


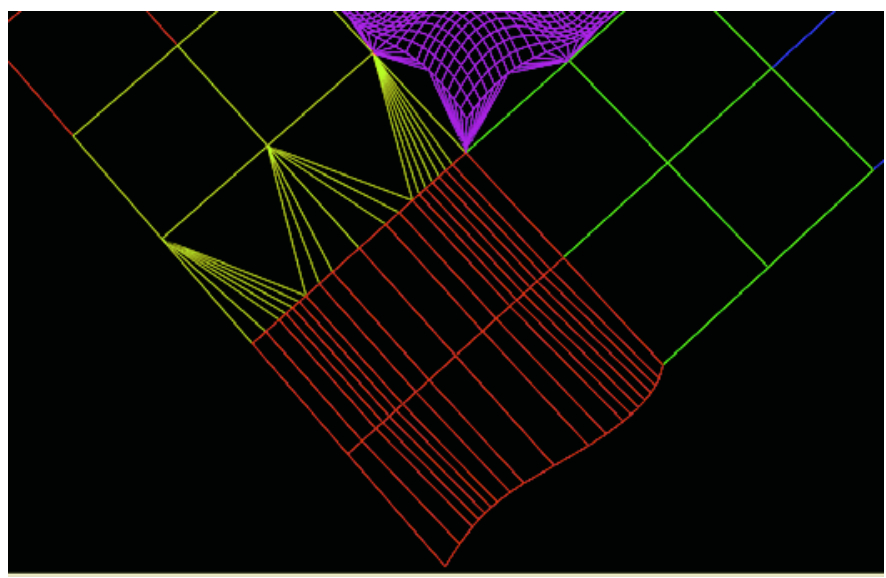

Fig. 4. Zippering In Action

We use zippering to prevent cracks from forming. Zippering leaves the interior of patches untouched, allowing these regions to be tessellated without concern for neighboring patches. To eliminate cracks between adjacent patches (as in Figure 3), the portion of a patch that is immediately in contact with an adjacent patch is carefully tessellated using a zipper-like configuration, so as to seamlessly move from a lower to higher level of tesselation. An example of zippering is shown in Figure 4. We tested the crack prevention algorithm on a large selection of objects, making sure that the method works on corner cases such as the fans of patches shown in figure 4.

\section{Performance Measurements}

In order to measure the performance of the algorithm, we tested our prototype on five dynamic scenes. Each scene comprised seven teapots that were spun along different elliptical paths. At any given moment, some teapots are close to the viewer, while others are far away.

All tests were performed on a Pentium 4 2.4Ghz machine with a GeForce4 MX440 video card. We realize that the performance testing is done on a software simulation of the Tesselator Unit architecture since none of the existent GPU's has one today. Therefore, the performance numbers are only indicative of the performance of the actual architecture. Nevertheless, it was immediately observed that the dynamic tessellation compares favorably with the fixed tesselation since it shows higher frame rates in most cases, as described below.

The quality of the rendering is improved as well, especially for the cases when the objects are very close to the viewer. We observed no disadvantages to our method as compared to the conventional method. We experimented with a scene that had a fixed tesselation of $64 \mathrm{k}$ triangles. By using the real time tesselation the number of triangles varied from a maximum of $16 \mathrm{k}$ (closest from the viewer) down to a few hundreds 


\begin{tabular}{|c|c|c|c|c|c|}
\hline \multicolumn{6}{|c|}{ RTT } \\
\hline \multirow[t]{2}{*}{$\mathrm{N}$} & \multirow[t]{2}{*}{$\begin{array}{c}\text { Triangles } \\
\text { Generated }\end{array}$} & \multicolumn{2}{|c|}{ Transform + Tessellate } & \multicolumn{2}{|c|}{$\begin{array}{c}\text { Transform }+ \text { Tessellate }+ \\
\text { Render }\end{array}$} \\
\hline & & milliseconds & fps & milliseconds & fps \\
\hline 0.5 & $29 \sim 34 \mathrm{~K}$ & 21.7 & 43.1 & 23.6 & 40.1 \\
\hline 0.7 & $24 \sim 28 \mathrm{~K}$ & 19.9 & 47.0 & 21.5 & 43.8 \\
\hline 1.0 & $24 \sim 26 \mathrm{~K}$ & 19.3 & 48.3 & 20.9 & 45.0 \\
\hline 2.0 & $24 \sim 26 \mathrm{~K}$ & 19.2 & 48.7 & 20.8 & 45.3 \\
\hline \multicolumn{6}{|c|}{ Offline Tessellation } \\
\hline \multirow{2}{*}{$\begin{array}{l}\text { Triangles } \\
\text { Per Patch }\end{array}$} & \multirow{2}{*}{$\begin{array}{c}\text { Triangles } \\
\text { Generated } \\
\text { Overall }\end{array}$} & \multicolumn{2}{|c|}{ Transform } & \multicolumn{2}{|c|}{ Transform + Render } \\
\hline & & milliseconds & fps & milliseconds & fps \\
\hline 128 & $28 \mathrm{~K}$ & 6.3 & $60+$ & 15.2 & $60+$ \\
\hline 512 & $115 \mathrm{~K}$ & 18.3 & 51.3 & 30.1 & 32.0 \\
\hline 2048 & $459 \mathrm{~K}$ & 66.7 & 14.5 & 81.9 & 11.9 \\
\hline
\end{tabular}

Fig. 5. Performance Measurements: Averages

(farthest from the viewer) clearly demonstrating the compression capabilities of our method. Since the main performance savings in our architecture will come from the AGP/PCIX bus bandwidth reduction we wanted to verify that our approach does not create a bottleneck inside the GPU. We obtained ample proof that this is not the case by trying many scenarios that allow for complex animations of flocks of objects in the context of a variable viewpoint. We have made several movies of our animations as well as a menu driven interactive demo.

The "RTT" entry represents our real time tesselation method. We separated the measurements into transform+tesselate only vs. transform+tesselate+render. The reason is that we wanted to measure the exact effects of the tesselation by comparison with conventional offline tesselation. In a real GPU there would be a tesselator unit stage, so that the effects of tesselation on execution time would be hidden by the fact that the GPU is pipelined. We rendered the same animation four different times, each time at a different criterion of subdivision termination $(n=0.5, n=0.7, n=1, n=2)$. As $n$ (the fractional deviation of the planar approximation from the real surface, expressed in pixels) increases, the number of triangles generated from subdivision decreases and the speed increases.

We also implemented a feature to simulate the current standard rendering methods whereby models are tessellated offline and then sent to the GPU as sets of triangles. This is the "Offline Tesselation" entry at the bottom of the table. Each patch is tessellated uniformly to a user-defined number of triangles $(128,512$, or 2048). On every frame, each pre-calculated vertex is transformed from model space into world coordinates. The normal of each vertex is also appropriately transformed into world coordinates. Then the triangle is rendered directly.

\section{Conclusion}

We developed a new 3D graphics architecture using data compression to unclog the bus between the triangle server and the rendering engine. The data compression is achieved 
by replacing the conventional idea of a rendering engine that renders triangles with a rendering engine that will tessellate surface patches into triangles. Thus, the bus sends control points of the surface patches, instead of the many triangle vertices forming the surface, to the rendering engine.

\section{References}

1. Lien, S.L., Shantz, M., Pratt, V.R.: Adaptive forward differencing for rendering curves and surfaces. In: SIGGRAPH '87 Proceedings, ACM (1987) 111-118

2. Lien, S.L., Shantz, M.: Shading bicubic patches. In: SIGGRAPH '87 Proceedings, ACM (1987) 189-196

3. Moreton, H.P.: Integrated tesselator in a graphics processing unit. U.S. patent (2003) \#6,597,356.

4. Sfarti, A.: Bicubic surface rendering. U.S. patent (2003) \#6,563,501.

5. Lane, J.F., Carpenter, L.C., Whitted, J.T., Blinn, J.F.: Scan line methods for displaying parametrically defined surfaces. In: Communications of the ACM. Volume 23(1)., ACM (1980) 23-24

6. Forsey, D.R., Klassen, R.V.: An adaptive subdivision algorithm for crack prevention in the display of parametric surfaces. In: Proceedings of Graphics Interface. (1990) 1-8

7. Clark, J.H.: A fast algorithm for rendering parametric surfaces. In: Computer Graphics (SIGGRAPH '79 Proceedings). Volume 13(2) Special Issue., ACM (1979) 7-12

8. Moreton, H.P.: Watertight tesellation using forward differencing. In: Proceedings of the ACM SIGGRAPH/Eurographcs workshop on graphics hardware. (2001)

9. Chung, A.J., Field, A.: A simple recursive tesselator for adaptive surface triangulation. JGT 5(3) (2000)

10. Moule, K., McCool, M.: Efficient bounded adaptive tesselation of displacement maps. In: Graphics Interface 2002. (2002)

11. Boo, M., Amor, M., Dogget, M., Hirche, J., Strasser, W.: Hardware support for adaptive subdivision surface rendering. In: Proceedings of the ACM SIGGRAPH/Eurographics workshop on Graphics Hardware. (2001) 33-40

12. Hoppe, H.: View-dependent refinement of progressive meshes. In: Proceedings of the 24th annual conference on computer graphics and interactive techniques. (1997)

13. Sfarti, A.: System and method for adjusting pixel parameters by subpixel positioning. U.S. patent (2001) \#6,219,070.

14. Barsky, B.A., DeRose, T.D., Dippe, M.D.: An adaptive subdivision method with crack prevention for rendering beta-spline objects. Technical Report, UCB/CSD 87/384, Computer Science Division, Electrical Engineering and Computer Sciences Department, University of California, Berkeley, California, USA (1987)

15. Velho, L., de Figueiredo, L.H., Gomes, J.: A unified approach for hierarchical adaptive tesselation of surfaces. In: ACM Transactions on Graphics. Volume 18(4)., ACM (1999) 329-360

16. Kahlesz, F., Balazs, A., Klein, R.: Nurbs rendering in opensg plus. In: OpenSG 2002 Papers. (2002) 